\title{
Les tournesols adventices : un exemple d'évolution d'une mauvaise-herbe apparentée à une espèce cultivée - Synthèse des recherches menées sur les populations adventices de tournesol en France depuis 2004
}

\author{
Marie-Hélène MULLER ${ }^{1}$ \\ Marie ROUMET ${ }^{1}$ \\ Vincent LECOMTE ${ }^{2}$ \\ 1 INRA UMR AGAP, \\ Place Pierre Viala, \\ 34060 Montpellier, \\ France \\ $<$ Marie-Helene.Muller@supagro.inra. \\ $\mathrm{fr}>$ \\ ${ }^{2}$ CETIOM, \\ 6 chemin de la côte vieille, \\ 31450 Baziège, \\ France
}

Article reçu le 11 juillet 2012

Accepté le 30 juillet 2012

\begin{abstract}
Evolution of a crop-related weed: Synthesis of researches conducted on weedy sunflower populations in France since 2004

Weedy sunflowers have been officially reported in South-Western France in 2004. They display a combination of phenotypic traits of the wild and domesticated forms of the species and infest between 15 and 20\% of fields of sunflower crop in this area, although at variable levels. When the infestation is strong, it affects seed yield and oil quality. Molecular studies showed that weedy sunflowers most probably resulted from accidental crop-wild hybridization during the seed production process and from the introduction of the resulting hybrids into commercial seed lots. Multiple independent introductions were at the source of the number of infested fields observed nowadays. The temporal dynamics of the infestation of a field and the detailed conditions for its success remain largely unknown. The flowering synchrony between crops and weeds is substantial, even if a variable fraction of the weeds (15-55\%) flower completely outside the crop flowering period. Molecular survey of adult weedy plants and their progenies showed that crop-toweed gene flow occurs, at a rate that can reach $35 \%$ on average, at the peak of the crop flowering period. We draw perspectives on the durability of herbicide-tolerant sunflower varieties as a solution to control these weeds, and call for more studies tackling weed management from an evolutionary lens.
\end{abstract}

Key words: sunflower, weed evolution, weed management, population genetics, phenology

synthèse des recherches conduites sur ces populations depuis leur identification en France en 2004.

Ces recherches ont associé des membres d'un centre technique interprofessionnel et d'un laboratoire de génétique situé à l'interface de l'amélioration des plantes et de la biologie évolutive. En effet, comme les formes adventices de riz ou de betterave, les tournesols adventices ont la particularité d'appartenir à une espèce qui est elle-même cultivée et d'infester les champs de cette espèce. Cette double spécificité rend ces mauvaises herbes plus difficiles à contrôler. Mais elle en fait également des modèles intéressants pour la biolo- gie évolutive car elle pose la question de l'adaptation et de la différenciation de deux formes apparentées (i.e. la forme cultivée et les formes adventices) alors qu'elles poussent dans le même milieu.

Nous commencerons par présenter rapidement I'histoire du tournesol cultivé, sa domestication à partir du tournesol sauvage et les différentes étapes de son amélioration. Puis, nous présenterons la situation agronomique des tournesols adventices, les travaux qui ont permis de comprendre leur origine, et ceux qui se sont intéressés à l'importance des flux géniques en provenance des variétés cultivées. Enfin, nous dresserons des perspectives sur le potentiel évolutif de

Pour citer cet article : Muller MH, Roumet M, Lecomte V. Les tournesols adventices : un exemple d'évolution d'une mauvaise-herbe apparentée à une espèce cultivée - Synthèse des recherches menées sur les populations adventices de tournesol en France depuis 2004OCL 2012; 19(5) : 304-312. doi : 10.1684/ocl.2012.0465 
ces populations, notamment dans le contexte de la mise en culture de variétés tolérantes à des herbicides.

\section{Petite histoire du tournesol cultivé : domestication, amélioration, introductions, réintroductions}

L'espèce Helianthus annuus, comme l'ensemble du genre Helianthus est originaire du nord du continent américain (Seiler, Rieseberg, 1997). Elle y existe à l'état sauvage sur une large zone géographique allant du nord du Mexique au sud du Canada. Présente dans une grande diversité d'habitats et de conditions climatiques (Nooryazdan et al., 2010), elle se développe préférentiellement au sein de milieux perturbés par I'Homme (Seiler, Rieseberg, 1997), y compris dans les champs cultivés où elle est considérée comme une mauvaise herbe très nuisible (Encadré 1, Kane, Rieseberg, 2008).
La domestication du tournesol a d'abord été réalisée par les Indiens plus de 2000 ans av. J.C., dans une zone correspondant au centre-est des États-Unis (Blackman et al., 2011). Les premières modifications morphologiques ont affecté la ramification et la taille des graines (Putt, 1997). Les indiens consommaient les graines sous diverses formes, et en faisaient également des usages non alimentaires (cérémonies, teintures, Putt, 1997). Le tournesol cultivé a été introduit en Europe après les premiers voyages intercontinentaux du $\mathrm{XVI}^{\mathrm{e}}$ siècle, essentiellement comme plante ornementale. Une seconde histoire a commencé pour lui à partir du XIX siècle, avec le développement de la culture pour la production d'huile en Russie. La sélection a permis d'augmenter la teneur en huile des graines; des variétés riches en huile ont alors été diffusées en Europe puis à travers le monde, et notamment en Amérique du Nord. La dernière étape importante de son histoire correspond au développement de variétés hybride-F1 dans les années 1970, grâce à la découverte et l'exploitation de la stérilité-mâle cyto- plasmique. Le tournesol est actuellement cultivé dans le monde entier, essentiellement pour son huile, plus marginalement pour la consommation directe des graines, et, plus récemment, pour des usages industriels (biodiesels par exemple, Dominguez Brando, Sarquis, 2012).

Les différentes étapes de cette histoire ont conduit à une différenciation marquée entre les formes sauvages et domestiquées modernes de tournesol (figure 1): par opposition à la forme sauvage, les variétés cultivées actuelles sont non ramifiées et ont perdu la pigmentation anthocyanée dans les tiges, pétioles et capitules. Elles sont généralement plus précoces (même si une grande variabilité existe chez la forme sauvage), autocompatibles, c'est-à-dire capables de produire des graines en autofécondation, et produisent des graines plus grosses et plus riches en huile (Burke et al., 2002). Deux caractères particulièrement importants concernent la dormance et la dispersion spontanée des graines: les graines de la forme domestique ont un fort taux de germination en conditions optimales de température et d'humidité,

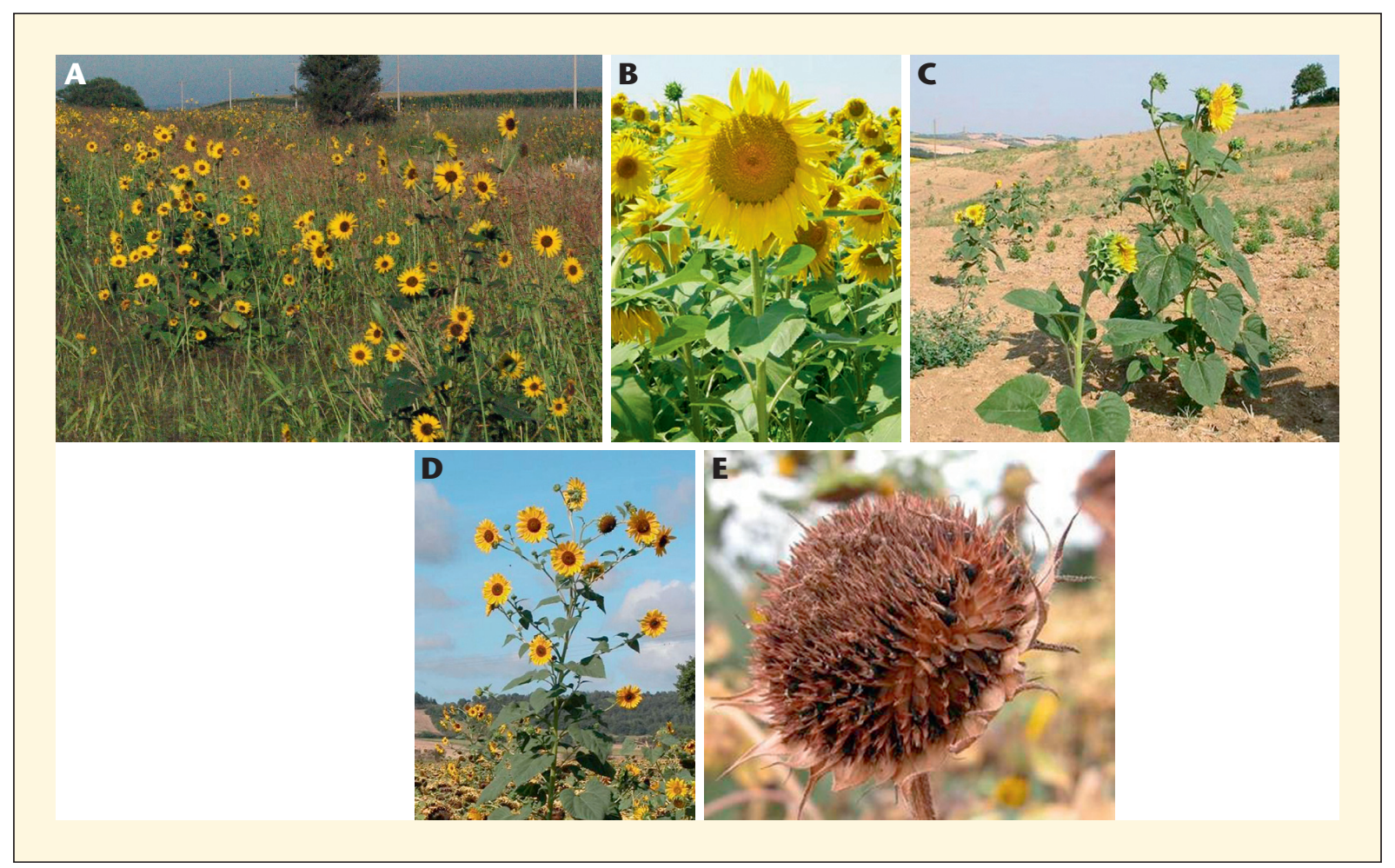

Figure 1. Illustrations de différents morphotypes au sein de l'espèce Helianthus annuus. A) Tournesols sauvages. B) Tournesol cultivé classique (champ à proximité de Toulouse). C) Repousses de tournesol cultivé dans une jachère (proximité de Toulouse). D) Tournesol adventice (champ à proximité de Toulouse). E) Capitule de tournesol adventice ayant perdu ses graines à maturité. 
alors que celles de la forme sauvage peuvent survivre sans germer plusieurs années dans la banque de graines. De même, alors que les graines ${ }^{1}$ restent sur le capitule de la forme cultivée, elles tombent quasiment toutes à maturité chez la forme sauvage (figure 1).

Outre les variétés conventionnelles, il est important de mentionner les variétés ornementales, vendues très communément dans les jardineries. Ces variétés présentent une grande diversité de morphologie, de taille, de couleur. Mais leurs schémas de sélection et leurs origines génétiques restent mal connus.

\section{Les populations adventices de tournesol : un nouveau problème pour le tournesol, identifié et suivi depuis 2004}

En Europe, la forme sauvage de l'espèce Helianthus annuus n'est théoriquement pas présente de façon naturelle. En bord de champs, dans les jachères, ainsi que dans les champs eux-mêmes, on observe couramment des repousses, issues de graines de tournesol cultivé perdues à la récolte. Ces plantes sont phénotypiquement proches de la forme domestiquée, même si quelques-unes sont ramifiées (ségrégation d'un allèle récessif de ramification présent à l'état hétérozygote dans les variétés hybrideF1) : en particulier, elles ne sont pas anthocyanées et leurs graines ne sont ni dormantes ni dispersées spontanément (figure 1). Les quelques prospections réalisées suggèrent qu'elles ne constituent pas un problème agronomique notable. Par opposition à d'autres repousses, comme le colza (Pessel et al., 2001), elles semblent ne former que des populations transitoires (i.e. les graines de l'année ne donnent pas naissance à des générations ultérieures, Ostrowski et al., 2010).

En 2004, des plantes morphologiquement originales par rapport aux repousses et aux variétés cultivées ont pour la première fois été identifiées de manière formelle en France, dans la région du

\footnotetext{
${ }^{1}$ Botaniquement, la structure dispersée est un akène (fruit indéhiscent), mais par souci de simplicité, nous utiliserons le terme graine dans le texte.
}

Lauragais (sud-ouest de la France). Elles se développent essentiellement à l'intérieur des parcelles cultivées en tournesol où elles atteignent des densités variables allant de quelques plantes sporadiques à une quinzaine de plantes par $\mathrm{m}^{2}$ (figure 2). Dans les populations qu'elles constituent, on rencontre toute une gamme de morphologies combinant des caractères de la forme domestique et de la forme sauvage (e.g. forte ramification, pigmentation anthocyanée). On y trouve donc aussi bien des plantes de type repousse et des plantes de type sauvage (figure 1), avec tous les intermédiaires possibles.

Lorsqu'elles sont en forte densité, ces plantes affectent fortement la culture du tournesol avec des pertes de rendement pouvant atteindre $50 \%$ (Muller et al., 2009). Les fortes densités ainsi que l'abondance de tiges vertes tard dans la saison rendent la récolte très difficile, voire impossible. L'effet sur la qualité de I'huile a été mesuré : la composition des graines à l'échelle d'un capitule cultivé n'est pas modifiée. En revanche, si on échantillonne les graines à la sortie de la moissonneuse-batteuse, la teneur en acide oléique (un critère déterminant pour la commercialisation des lots dans les filières correspondantes) est réduite du fait de mélange de graines cultivées et adventices à la récolte (Lecomte, 2007).

Des enquêtes kilométriques ont été menées par le CETIOM depuis 2005 afin d'évaluer l'ampleur du phénomène, d'abord à l'échelle de trois régions (Lauragais, Gers et Poitou-Charentes), puis sur l'ensemble des zones de production du tournesol du sud-ouest (figure 3, dans le reste de la France, le phénomène reste à ce jour limité). Elles consistent à suivre chaque année les mêmes axes routiers, et à noter le niveau d'infestation de parcelles de tournesol observées sur le parcours. Elles ont établi qu'entre 15 et $20 \%$ des parcelles de tournesol étaient concernées. Cette fréquence a peu varié pendant la période de suivi. Les niveaux d'infestation sont cependant hétérogènes. En 2011, 1 \% des parcelles ont été catégorisées

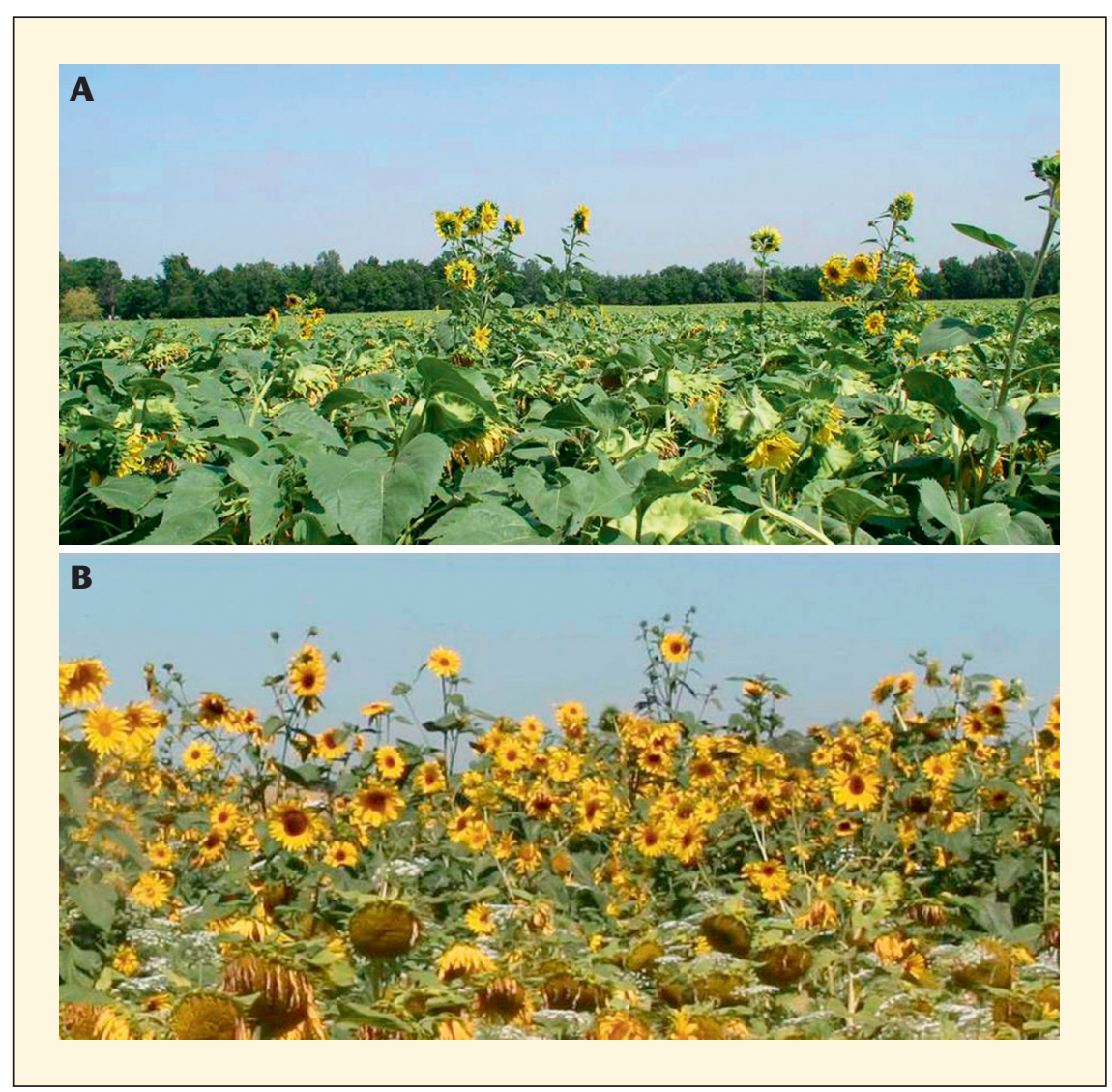

Figure 2. Situations contrastées d'infestation par des tournesols adventices (proximité de Toulouse). A) Champ faiblement infesté. B) Champ fortement infesté. 


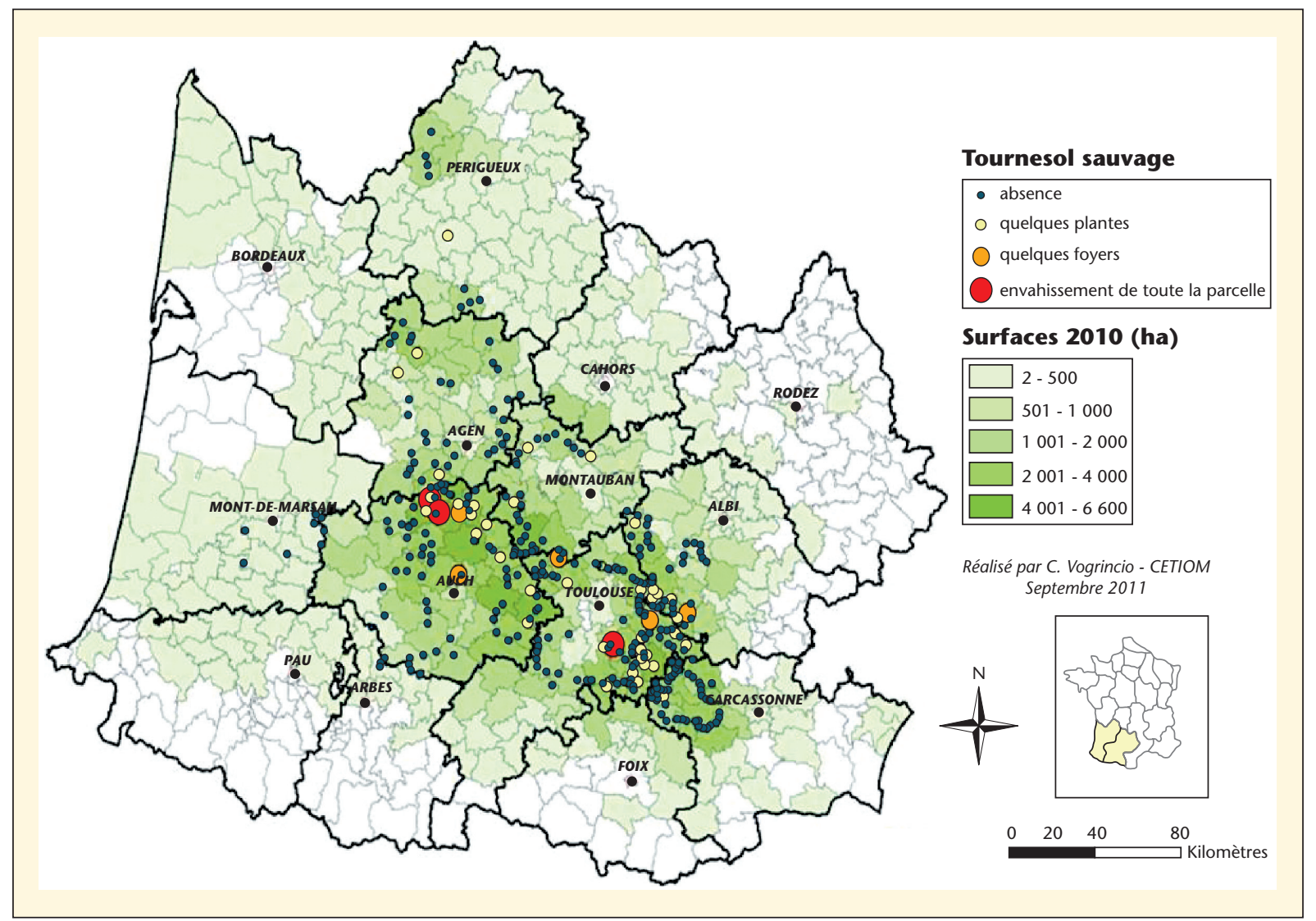

Figure 3. Localisation et niveau d'infestation des parcelles observées lors de l'enquête kilométrique 2011. Enquête réalisée entre le 29/07/2011 et le 19/08/2011 sur 350 parcelles (Source: CETIOM, suivi BSV). Tournesol "sauvage" est la terminologie adoptée par le CETIOM dans sa communication sur les tournesols adventices.

comme fortement infestées, c'est-à-dire qu'elles présentaient de fortes densités de plantes adventices sur la quasi-totalité de la parcelle (figure 3).

Des suivis fins ont étés réalisés par le CETIOM de 2005 à 2010 sur une dizaine de sites; chaque site était constitué par un petit groupe de parcelles centrées autour d'une parcelle identifiée comme fortement infestée. Cette étude a montré qu'à partir d'un niveau d'infestation moyen (quelques foyers), la population adventice était présente durablement. Les taux de transition $d^{\prime}$ un niveau d'infestation à un autre, estimés sur les 6 années d'étude, ont permis de simuler la progression du phénomène au fil des années : après 10 rotations tournesol/blé (soit vingt ans), un tiers des parcelles atteint un niveau d'infestation moyen.

Des entretiens ont été menés en 2005 et 2006 auprès d'agriculteurs confrontés au phénomène dans le Lauragais. II en ressort que les premiers cas remontent au début des années 1990. Mais depuis le début des années 2000 les tournesols adventices sembleraient prendre plus d'importance. Aucun facteur majeur favorisant leur présence dans une parcelle $\mathrm{n}^{\prime}$ a été identifié, même si quelques éléments semblent augmenter le risque :

- les sols profonds par opposition aux sols superficiels ;

- les rotations courtes avec tournesol (généralement un an sur deux dans le Lauragais) ;

- la présence, une année donnée, de pertes abondantes de graines lors la récolte du tournesol.

Dès 2005, le CETIOM a mis en place une information auprès des professionnels, afin notamment de préconiser un repérage, et donc une élimination précoce des adventices dans les parcelles. Cette méthode apparaissait comme la seule méthode efficace pour éliminer ou au moins contenir ces populations, avant le développement de variétés tolérantes à des herbicides dont il sera question plus loin.
Ces premiers travaux ont donc mis en évidence que ces populations adventices constituaient une forme originale de tournesol qui se distinguait à la fois des populations sauvages américaines et des variétés cultivées. Elles causent des problèmes réels aux agriculteurs, et en ont déjà amené certains à abandonner la culture du tournesol. Si leur prévalence devait augmenter, elles pourraient menacer cette culture à une plus large échelle.

\section{À I'origine des populations adventices françaises et espagnoles : des croisements non désirés dans les champs de production de semences}

Des études moléculaires ont été conduites sur un échantillon de populations adventices françaises et espagnoles (Muller et al., 2011). Toutes les plantes 
adventices analysées présentaient les marqueurs caractéristiques du génome mitochondrial des variétés hybride-F1 (cytoplasme mâle-stérile). L'ADN mitochondrial étant transmis strictement par voie maternelle chez le tournesol, ce résultat implique que les populations adventices ont à leur origine des plantes-mères issues de variétés cultivées. L'analyse du génome nucléaire par 16 marqueurs microsatellites, a de son côté révélé une diversité beaucoup plus importante que celle présente dans les variétés: de 4 allèles par locus en moyenne dans un pool représentatif de variétés cultivées, la richesse allélique s'élève à plus de 8 allèles par locus dans le pool de populations adventices françaises analysées, et plus de 10 pour les populations espagnoles (figure 4A). Plus précisément, tous les allèles présents dans les variétés sont retrouvés dans les populations adventices, et en fréquence similaire. Mais les populations adventices présentent de nombreux allèles supplémentaires, appelés "allèles originaux", chacun en fréquence faible (figure $4 B$, pour un exemple à un locus). De leur côté, les populations de repousses ne contiennent que les allèles présents dans les variétés cultivées. Les variétés ornementales présentent certes des allèles originaux, mais leur diversité ne permet pas d'expliquer toute celle observée dans les populations adventices. Enfin, une étude sur un large échantillon de populations sauvages américaines (données non publiées) a révélé une très large diversité, incluant en particulier, tous les allèles originaux présents dans nos populations adventices.

Le scénario le plus vraisemblable quant à l'origine de ces populations impliquerait donc la pollinisation de lignées maternelles par des formes sauvages poussant à proximité des champs de production de semences. Des hybrides sauvagescultivés auraient ainsi été introduits dans des lots de semences vendus aux agriculteurs, et donc dans les parcelles semées avec ces lots de semences. Lors de la première année de culture, les contaminants seraient présents sur les rangs. Les graines produites par ces premiers hybrides, et éventuellement dispersées, donneraient alors naissance à des plantes entre les rangs, point de départ de l'invasion d'une parcelle (figure 5). Des origines similaires ont été démontrées pour d'autres mauvaises

\section{A}

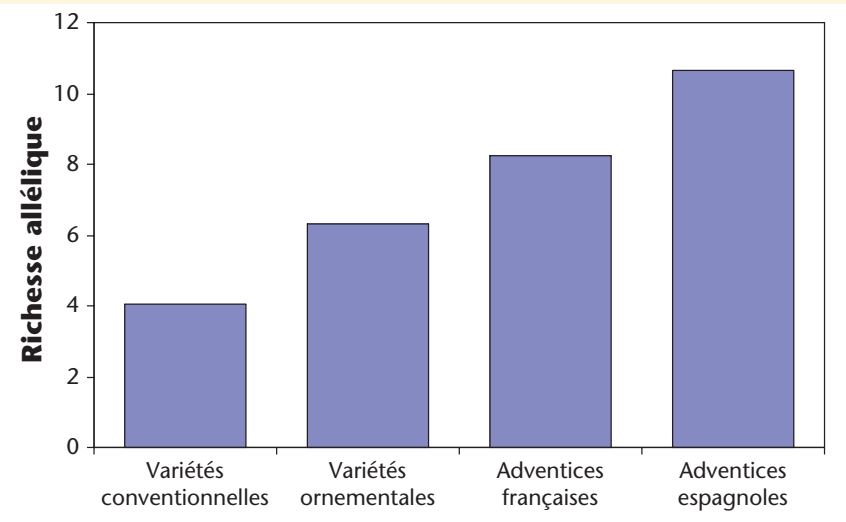

B

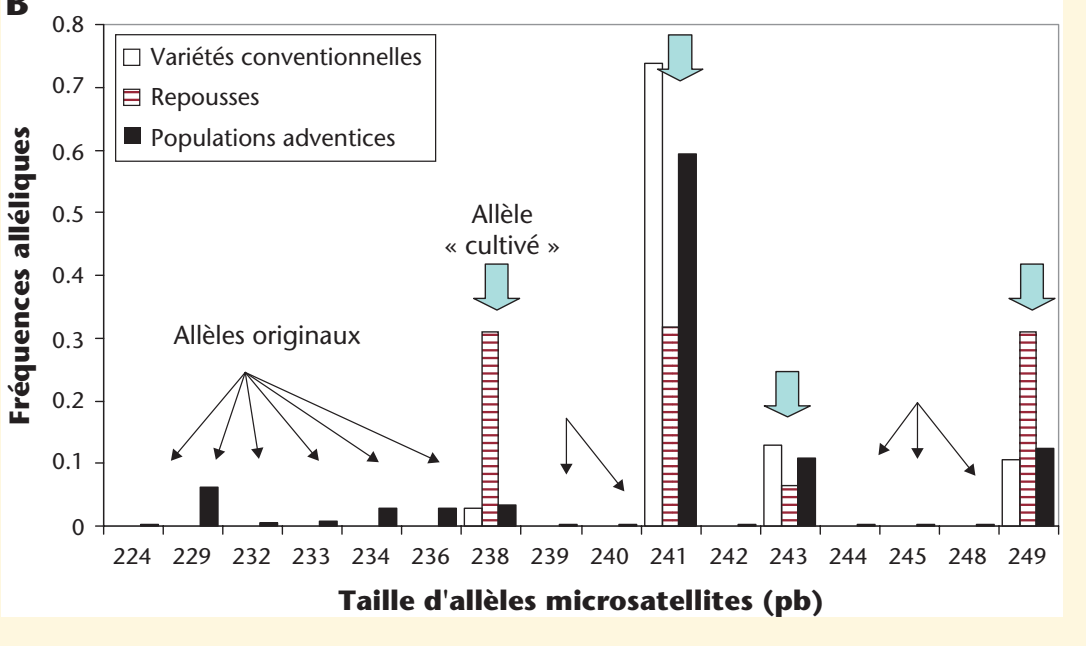

Figure 4. Deux résultats marquants de l'analyse de la diversité génétique sur des marqueurs microsatellites. A) Richesse allélique par groupe de populations: nombre moyen d'allèles par locus, standardisé pour une même taille d'échantillon par groupe. B) Distribution des fréquences alléliques dans 3 groupes, pour le locus ORS887. Les fleches vertes pointent les allèles présents dans les variétés cultivées, et partagés avec les populations adventices. Les autres fleches pointent les alleles dits " originaux ".

herbes apparentées à une espèce cultivée (par exemple, les betteraves adventices, Boudry et al., 1993).

Les champs de production de semences à l'origine des premières introductions devaient se trouver dans une zone où des formes sauvages sont présentes, c'est-à-dire probablement en Amérique du Nord. Cependant, dans la mesure où il existe des formes adventices et sauvages naturalisées ailleurs dans le monde (encadré 1), la contamination peut désormais se produire dans une aire géographique plus large. Ces dernières années, nous avons régulièrement observé des plantes d'aspect hybride sur les rangs de champs de tournesol, ce qui suggère que les semences commerciales sont encore à l'origine de nouvelles infestations. En particulier, lors des enquêtes kilométriques 2010 et 2011, dans $40 \%$ des parcelles présentant des tournesols adventices, ceux-ci ont été observés sur le rang à des densités inférieures à 5 pieds par ha. Ce phénomène est très préoccupant. En effet, aucune gestion durable contre les tournesols adventices ne pourra être mise en œuvre avec efficacité tant que des lots de semences contaminés par des formes adventices seront mis sur le marché.

Une question émerge de ce scénario : les différentes parcelles infestées sont-elles issues d'introductions indépendantes, ou bien y a-t-il eu diffusion de parcelles en parcelles après une première invasion? Plusieurs types de données 


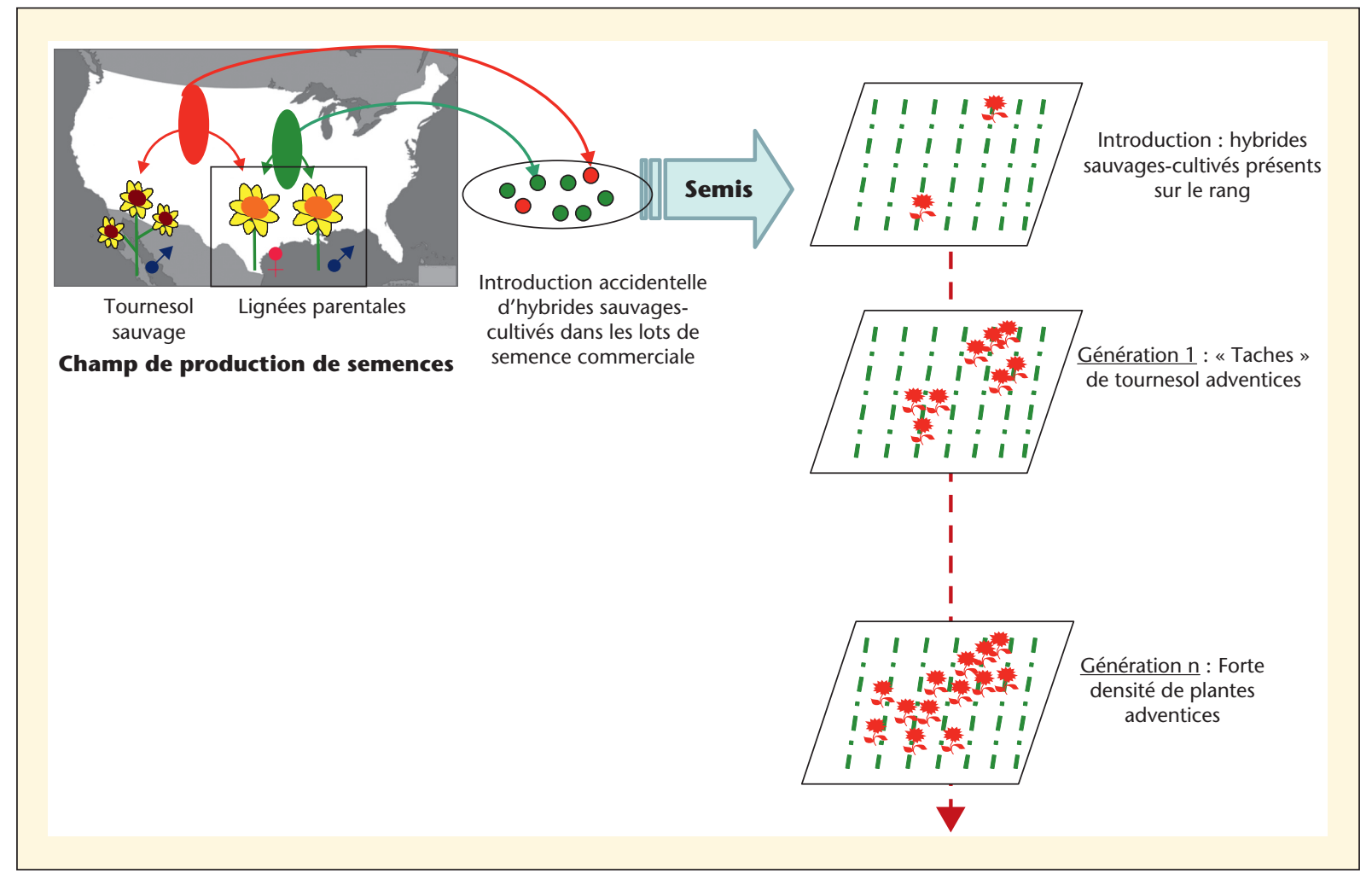

Figure 5. Scénario proposé pour l'origine des populations adventices.

collectées dans le Lauragais permettent de répondre. D'une part, les parcelles infestées se retrouvent sur l'ensemble du territoire, de façon apparemment aléatoire (i.e. pas d'agrégation de parcelles plus ou moins infestées). Souvent, des parcelles très infestées jouxtent des parcelles libres de toute infestation. D'autre part, l'analyse de la structuration géographique de la diversité génétique montre une répartition elle-aussi au hasard des allèles originaux, et donc, là encore, une absence de trace de foyers d'introduction autour de laquelle les populations adventices se seraient diffusées.

L'ensemble de ces résultats suggère que les parcelles infestées fonctionnent

\section{Encadré 1}

\section{Les formes spontanées de tournesol à travers le monde}

D'autres exemples de formes adventices de tournesol existent à travers le monde. En particulier, l'espèce est considérée aux États-Unis comme une mauvaise herbe nuisible affectant des cultures de printemps (soja, maïs) avec un impact économique considérable (Kane, Rieseberg, 2008). Dans ce cas, ce serait la forme sauvage qui aurait colonisé l'agro-écosystème et aurait évolué en mauvaise-herbe. Des formes mauvaises-herbes existent également en dehors de la zone d'origine de l'espèce, notamment en Italie, Espagne et Europe de l'Est. À I'exception de l'Espagne où nous avons mené des études en parallèle, aucune donnée précise n'a été publiée sur ces populations. Enfin, la forme sauvage $\mathrm{d}^{\prime}$ Helianthus annuus a été introduite volontairement ou non dans différentes parties du globe donnant lieu dans certains cas à des populations naturalisées. C'est le cas en Argentine où l'introduction de formes sauvages dans les années 1960 (comme plante fourragère) a conduit à des populations installées, parfois très grandes (Cantamutto et al., 2008). Leur écologie est similaire à la forme américaine : milieux perturbés, bords de route/de champ. Ces formes sont retrouvées parfois dans les parcelles, mais à quelques rares exceptions près, elles ne nuisent pas à l'agriculteur. essentiellement indépendamment les unes des autres et qu'il y aurait donc eu un grand nombre d'introductions plutôt que des diffusions autour de quelques parcelles infestées. Des diffusions ponctuelles ne sont pas exclues; cependant elles ne permettent pas d'expliquer à elles seules l'ampleur actuelle du phénomène.

Si l'hypothèse d'introduction ne soulève actuellement plus de doute, on n'a en revanche jamais suivi l'invasion d'une parcelle depuis ses premiers stades. Le scénario proposé (figure 5) conserve donc de nombreuses inconnues. Notamment, dans quelle mesure une introduction donnée est-elle suffisante pour conduire à une forte infestation ? Quel rôle jouent le nombre d'hybrides sauvages-cultivés initialement introduits et leurs traits phénotypiques? Des introductions récurrentes sont-elles nécessaires pour enrichir le pool génétique initialement introduit? Des données plus récentes (Roumet et al., soumis) ont révélé une richesse en allèles originaux plus importante au sein de populations de parcelles fortement infestées par rapport à des populations de parcelles faiblement infestées. Mais 
les données restent trop fragmentaires (6 populations analysées seulement) pour pouvoir conclure sur un lien entre forte diversité génétique introduite et "succès" de l'invasion.

Une autre question concerne la vitesse de progression de l'infestation: les suivis fins $n^{\prime}$ ont pas révélé d'évolution importante du niveau d'infestation des parcelles. Cependant ils n'ont été conduits que sur 6 ans (soit seulement 3 générations d'adventices vu la durée des rotations qui est généralement de deux ans). Par ailleurs, la dynamique de croissance des populations a pu être affectée par des événements exceptionnels: des automnes secs peuvent par exemple avoir empêché les habituelles levées post-récolte du tournesol et ainsi contribué à l'enrichissement de la banque de graines, entraînant une explosion démographique. II nous est donc encore impossible de faire des inférences sur le nombre de cycles de culture suffisant pour aboutir à une parcelle fortement infestée à partir de l'introduction des premiers hybrides.

Résorber les inconnues existantes dans notre scénario nécessiterait de réaliser une approche comparative sur un plus grand nombre de populations et un suivi sur une plus grande échelle de temps. Plutôt que de mener ce type d'étude, nous avons fait le choix de nous intéresser aux forces évolutives à l'œuvre dans la colonisation d'une parcelle par une population adventice, et ce, en nous concentrant sur un nombre restreint de populations. Par force évolutive, nous entendons ce qui a un effet sur la variabilité génétique d'une population (sélection, migration, mutation, dérive génétique). À terme, la compréhension de ces mécanismes peut permettre de dégager les facteurs qui font le succès d'une invasion. Nous nous sommes intéressés en premier lieu aux flux géniques entre les variétés cultivées et les populations adventices.

\section{Ensemble dans la même parcelle : les tournesols adventices reçoivent régulièrement des gènes des variétés cultivées, et beaucoup. . .}

Les flux géniques entre les variétés cultivées et les populations adventices peuvent avoir des effets antagonistes sur l'évolution de ces populations. D'une part, s'ils sont forts dans les premières générations, ils peuvent apporter de façon récurrente des caractères de type "Cultivé » (notamment l'absence de dormance et de dispersion des graines) et constituer ainsi un frein empêchant une population adventice de se différencier de la forme cultivée. Mais réciproquement, ces flux géniques peuvent leur apporter des caractères intéressants, susceptibles d'augmenter leur nuisibilité : c'est notamment le cas lorsque les variétés cultivées sont tolérantes à des herbicides.

Un certain nombre d'éléments nous laissaient penser que ces flux de gènes étaient fort probables: synchronie partielle de floraison entre variétés cultivées et tournesols adventices, appartenance à la même espèce, hybridation sauvage-cultivé à l'origine des populations adventices. L'objectif des études menées sur le sujet était donc de ne pas se contenter de répondre par oui ou par non à une question sur l'existence de flux de gènes, mais d'acquérir une vision intégrée et quantitative, nécessaire pour comprendre leur impact sur l'évolution des populations.

Dans une première approche, nous avons quantifié les potentialités de croisements, via la synchronie de floraison entre les deux formes. Pour cela, au sein de 6 parcelles plus ou moins infestées, nous avons déterminé la période de floraison du champ cultivé et suivi la floraison d'un échantillon représentatif de la population adventice (début et fin de floraison, ainsi qu'observation bi-hebdomadaire du nombre de capitules en fleur par plante). Ce travail a permis de décrire la variation de la surface de capitules adventices en fleur au cours du temps, et d'estimer le pourcentage de cette surface totale qui fleurissait pendant la floraison du champ cultivé. Cette valeur a été nommée "recouvrement de floraison ". Elle est d'au moins $25 \%$ et variable d'une parcelle à l'autre (jusqu'à $57 \%$, figure 6). Au sein des populations, le recouvrement varie entre individus: selon les parcelles, de 15 à $55 \%$ des individus, essentiellement les plus tardifs, fleurissent de façon complètement décalée par rapport au champ cultivé.

Les plantes phénotypées ont également été génotypées pour 15 marqueurs microsatellites. L'analyse conjointe du génotype d'une plante et de sa période de floraison a montré que la diversité génétique de la population était "structurée dans le temps ". Concrètement, les plantes précoces - et donc plus synchrones avec les variétés cultivées - sont génétiquement plus proches des variétés cultivées que les plantes plus tardives. Comme les marqueurs utilisés ne sont pas liés à des gènes impliqués dans la phénologie de floraison, ce résultat montre que des flux géniques ont lieu de façon récurrente entre les variétés cultivées et les populations adventices. Comme les individus à floraison précoce sont soumis à des flux de gènes plus

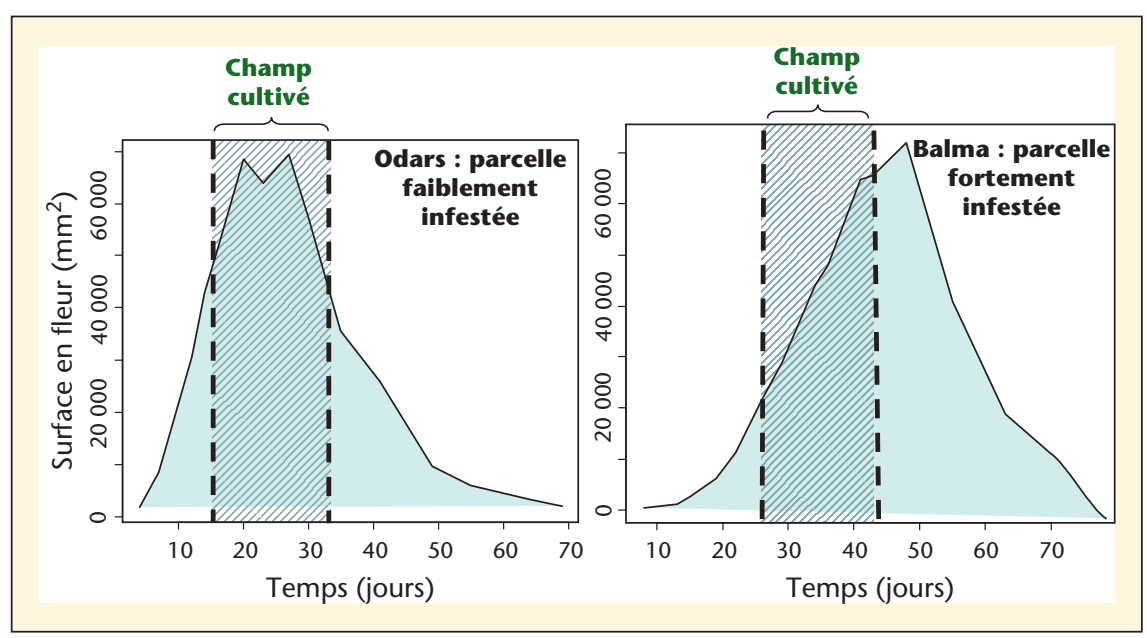

Figure 6. Recouvrement de floraison. Exemple de résultat obtenu dans deux parcelles différant pour leur niveau d'infestation. La courbe représente la variation de la surface adventice en fleur au cours du temps. Le recouvrement de floraison a été calculé comme la proportion de surface de fleur ayant fleuri pendant la floraison du champ cultivé (zone hachurée). 

cultivées que les tardifs, ceci conduit à une différenciation au sein même des populations adventices (Roumet et al., soumis).

Dans une deuxième approche, nous avons utilisé les mêmes marqueurs microsatellites pour génotyper des descendants de plantes adventices, afin d'estimer le taux de pollinisation de ces plantes par la variété cultivée. Ces plantes adventices ont été choisies au sein d'une des 6 populations évoquées ci-dessus, et leur date de floraison était connue. Pour des plantes fleurissant pendant le pic de croisement atteignent en moyenne $35 \%$. Ils décroissent ensuite rapidement pour atteindre zéro après la fin de floraison du champ (Roumet et al., en préparation, figure 7). Le taux moyen de croisement à l'échelle de la population atteint $11 \%$. Ce taux a plusieurs implications. D'une part, d'un point de vue évolutif, un tel niveau de flux génique est fort : s'il est récurrent sur plusieurs générations, il est suffisant pour homogénéiser rapidement des populations (Haygood et al., 2003). Cependant, au vu du rapport de force démographique (7 plantes cultivées pour 1 plante adventice en moyenne sur la parcelle, plus si l'on considère seulement les plantes adventices fleurissant pendant la floraison du champ cultivé), ce taux nous paraît relativement faible. II semble indiquer que les plantes adventices importants en provenance des variétés floraison du champ cultivé, les taux de

sont pollinisées préférentiellement par d'autres plantes adventices plutôt que par des plantes cultivées. Ces croisements non aléatoires entre individus au sein d'une même parcelle pourraient notamment résulter du comportement des pollinisateurs.

Les flux géniques existent, ont existé et structurent les populations adventices. Les taux de croisements que nous avons estimés ne correspondent qu'à la première étape de ces flux géniques: la dispersion du pollen. Pour aller plus loin dans la caractérisation des flux géniques et de leur impact évolutif, il sera nécessaire de quantifier les pressions de sélection s'exerçant sur les traits phénotypiques, et en particulier de s'intéresser à la valeur sélective des hybrides formés par rapport à celle de descendants de croisements entre plantes adventices.

\section{Quel est I'avenir de ces populations? Perspectives sur les méthodes de lutte et de contrôle}

Les mauvaises herbes constituent un exemple de colonisation d'un nouvel écosystème (I'agro-écosystème) et d'adaptation à celui-ci ; cependant leur analyse sous le prisme de l'évolution a longtemps été négligée. D’un point de vue fondamental comme appliqué, il

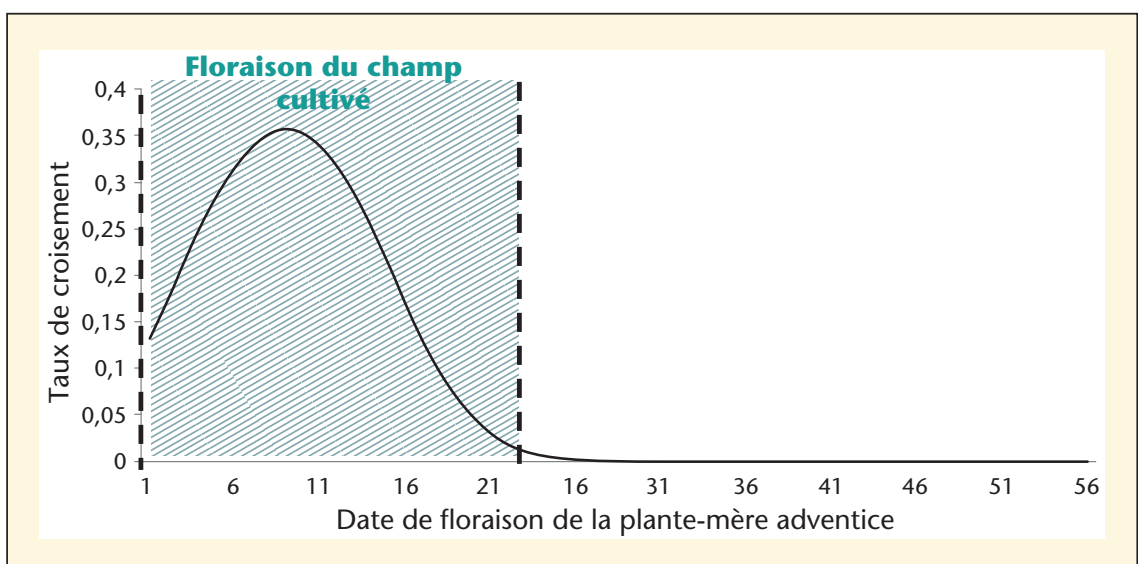

Figure 7. Taux de croisement par la variété en fonction de la date de floraison de la plantemère adventice. La courbe a été obtenue grâce à une approche prenant en compte (i) les données de génotypage de plantes adventices, de leurs descendants et de la variété cultivée, (ii) les dates de floraison des plantes adventices et du champ cultivé, et (iii) une modélisation de la présence de pollen "cultivé" dans le nuage pollinique au cours du temps. Ce type d'approche permet d'intégrer toutes les données pour prédire le taux de croisement attendu en fonction de la date de floraison d'une plante adventice. La date de floraison est reportée en jours depuis le début de la floraison du champ cultivé. apparaît important de décrire le fonctionnement de ces populations pour mettre en évidence les mécanismes régissant leur succès de colonisation et d'invasion d'un agro-écosystème. Pour le moment, les études menées sur les populations adventices de tournesol ont permis de montrer qu'elles proviennent de l'introduction accidentelle d'hybrides sauvage-cultivé, et que leur évolution est influencée par les flux de gènes en provenance des variétés cultivées. Mais nous ne pouvons pas encore tirer des conclusions précises sur (i) les caractéristiques de l'introduction favorisant l'invasion d'une parcelle (nombre et caractères des individus introduits) et (ii) le potentiel d'évolution au cours des générations (propension pour ces populations de devenir plus invasives et plus difficiles à contrôler). Dans quelle mesure de telles études peuvent-elles fournir des éléments permettant de les contrôler, de limiter leur diffusion et leur impact négatif ? Quelles préconisations peut-on donner quant à leur gestion ? Peut-on faire des prédictions sur leur évolution dans différentes situations?

Ces questions peuvent être abordées dans le cas particulier de la mise en culture de variétés tolérantes à des herbicides. Jusqu'à récemment, une gamme de méthodes était préconisée pour contenir l'infestation, la plus efficace étant le repérage précoce et I'arrachage systématique des plantes adventices dans les premiers stades d'une infestation. La mise sur le marché depuis 2010 de variétés tolérantes à des herbicides est susceptible d'apporter une solution plus simple et efficace. Mais la présence de flux géniques pose la question de la durabilité de cette solution. La résistance utilisée dans les variétés actuelles est monogénique et apporte un avantage évident aux plantes adventices qui la possède. Les taux de croisements que nous avons mis en évidence sont extrêmement forts pour un gène présentant une telle valeur sélective (Haygood et al., 2003).

Pour limiter le transfert de la tolérance herbicide aux formes adventices, et ainsi préserver un bon niveau d'efficacité de cette technique, le CETIOM a mis en avant des mesures d'accompagnement, et a bien insisté auprès des professionnels sur l'importance de leur strict respect (Duroueix, Leflon, 2009) : (i) ne laisser aucune zone non traitée dans la parcelle 
en culture ; (ii) à floraison du tournesol cultivé, détruire les tournesols adventices restant dans la parcelle et ceux éventuellement présents dans zones jouxtant la parcelle (bandes enherbées, coins de parcelles); (iii) éviter les situations où les parcelles cultivées en tournesols tolérants aux herbicides jouxtent des parcelles cultivées en tournesol classique, infestées, même faiblement, par des tournesols adventices. De façon générale, la solution herbicide est attendue d'autant plus efficace et durable que les tournesols adventices sont détruits dès leur apparition dans la parcelle.

Des suivis réalisés en 2010 chez les agriculteurs exposés aux tournesols adventices ont montré que les mesures préconisées n'étaient encore pas assez prises en compte. Nous avons en particulier observé des bosquets de tournesols adventices en fleur en bordure ou au sein (zones non traitées avec I'herbicide) d'une parcelle cultivée par un tournesol tolérant. La sensibilisation doit donc être poursuivie dans la durée afin d'éviter des transferts de la tolérance herbicide vers le tournesol adventice: pour un agriculteur ne suivant pas les conseils de lutte durable, la parcelle se retrouverait au bout de seulement deux ans (cas d'une rotation courte tournesol - blé) en présence de tournesols adventices tolérants à I'herbicide.

De façon plus générale, l'enseignement de la biologie évolutive est que le système est dynamique: la mise en œuvre d'une méthode de lutte est susceptible d'entraîner une réponse évolutive de la mauvaise herbe, lui permettant d'échapper à cette méthode de lutte. La réponse sera plus ou moins rapide en fonction de la méthode (intensité de la pression de sélection), et des caractéristiques des populations (taille, diversité génétique pour les caractères impliqués dans la réponse, importance des flux géniques, Neve et al., 2009). Par exemple, une forte dormance des graines est classiquement interprétée comme une réponse aux pratiques de labour et aux stratégies de faux semis (Clements et al., 2004).

La façon dont les connaissances sur la dynamique évolutive des mauvaises herbes aident à mettre en place des méthodes de lutte est un champ de recherche en plein développement (Neve et al., 2009). II s'est jusqu'à maintenant surtout concentré sur l'évolution des résistances aux herbicides (pression de sélection simple et forte, caractère monogénique), mais il apparaît maintenant important de s'intéresser à des caractères plus complexes. De telles études peuvent être la clé, en lien avec d'autres disciplines, pour contrôler de la façon la plus réfléchie possible les populations de mauvaises herbes.

Remerciements. Nous remercions toutes les personnes qui ont participé aux différents volets de cette étude, depuis la collecte des données de terrain et de laboratoire, jusqu'aux nombreuses discussions qui ont contribué à l'enrichir et à l'éclairer.

\section{Conflits d'intérêts : aucun}

\section{RÉFÉRENCES}

Blackman BK, Scascitelli M, Kane NC, et al. Sunflower domestication alleles support single domestication center in eastern North America. P Natl Acad Sci USA 2011 ; 108 : 14360-5.

Boudry P, Mörchen M, Saumitou-Laprade P, Vernet $P$, Van Dijk H. The origin and evolution of weed beets: consequences for the breeding and release of herbicide-resistant transgenic sugar beets. Theor Appl Genet 1993 ; $87: 471-8$.

Burke JM, Tang S, Knapp SJ, Rieseberg LH. Genetic analysis of sunflower domestication. Genetics 2002 ; 161 : 1257-67.

Cantamutto M, Poverene M, Peinemann N. Multi-scale analysis of two annual Helianthus species naturalization in Argentina. Agr Ecosyst Environ 2008 ; 123 : 69-74.

Clements DR, DiTommaso A, Jordan N, et al. Adaptability of plants invading North American cropland. Agr Ecosyst Environ 2004 ; 104 : 379-98.

Dominguez Brando J, Sarquis AV. Challenges for the sunflower oil market for 2020. Proceedings of the $18^{\text {th }}$ International Sunflower Conference, Mar del Plata, Argentina, 2012.

Duroueix F, Leflon M. Tournesols sauvages. Pour conserver une efficacité durable. Perspectives Agricoles 2009 ; 353 : 24-5.
Haygood R, Ives AR, Andow DA. Consequences of recurrent gene flow from crops to wild relatives. P Roy Soc London 2003 ; 270 : 1879 86.

Kane NC, Rieseberg LH. Genetics and evolution of weedy Helianthus annuus populations : adaptation of an agricultural weed. $\mathrm{Mol}$ Ecol 2008 ; 17 : 384-94.

Lecomte V. Tournesol "sauvage": une nouvelle adventice très concurrentielle du tournesol. Perspectives Agricoles 2007 ; 340 : 60-2.

Muller MH, Delieux F, Fernandez-Martinez $\mathrm{JM}$, et al. Occurrence, distribution and distinctive morphological traits of weedy Helianthus annuus L. populations in Spain and France. Genet Resour Crop Ev 2009 ; 56 : 869-77.

Muller M-H, Latreille M, Tollon C. The origin and evolution of a recent agricultural weed : population genetic diversity of weedy populations of sunflower (Helianthus annuus L.) in Spain and France. Evol Appl $2011 ; 4$ : 499514.

Neve P, Vila-Aiub M, Roux F. Evolutionarythinking in agricultural weed management. New Phytol 2009 ; 184 : 783-93.

Nooryazdan H, Serieys H, Bacilieri R, David J, Bervillé A. Structure of wild annual sunflower (Helianthus annuus L.) accessions based on agro-morphological traits. Genet Resour Crop Ev 2010 ; 57 : 27-39.

Ostrowski MF, Rousselle Y, Tsitrone A, et al. Using linked markers to estimate the genetic age of a volunteer population : a theoretical and empirical approach. Heredity $2010 ; 105$ : 358-69.

Pessel FD, Lecomte J, Emeriau V, et al. Persistence of oilseed rape (Brassica napus L.) outside of cultivated fields. Theor Appl Genet 2001 ; 102 : 841-6.

Putt ED. Early history of sunflower. In : Sunflower technology and production (ed. Schneiter AA), pp. 1-19. ASA, CSSA, SSSA, Madison, Wisconsin, USA, 1997.

Roumet M, Noilhan C, Latreille M, David J, Muller $\mathrm{M}-\mathrm{H}$. How to escape from crop-toweed gene flow: phenological variation and isolation-bytime within weedy sunflower populations. (Soumis à New Phytologist).

Seiler GJ, Rieseberg LH. Systematics, Origin and Germplasm resources of the wild and domesticated sunflower. In : Sunflower technology and production (ed. Schneiter AA). ASA, CSSA, SSSA, Madison, Wisconsin, USA, $1997: 21-65$. 